\title{
ARTICLE
}

Received 21 Jun 2013 | Accepted 18 Nov 2013 | Published 16 Dec 2013 DOl: 10.1038/ncomms3968

\section{Dampened regulates the activating potency of Bicoid and the embryonic patterning outcome in Drosophila}

Junbo Liu' $\&$ Jun $M a^{1,2}$

The Drosophila morphogen gradient of Bicoid (Bcd) initiates anterior-posterior (AP) patterning; however, it is poorly understood how its ability to activate a target gene may have an impact on this process. Here we report an F-box protein, Dampened (Dmpd) as a nuclear cofactor of Bcd that can enhance its activating potency. We establish a quantitative platform to specifically investigate two parameters of a Bcd target gene response, expression amplitude and boundary position. We show that embryos lacking Dmpd have a reduced amplitude of Bcd-activated hunchback ( $h b)$ expression at a critical time of development. This is because of a reduced Bcd-dependent transcribing probability. This defect is faithfully propagated further downstream of the AP-patterning network to alter the spatial characteristics of evenskipped (eve) stripes. Thus, unlike another Bcd-interacting F-box protein Fate-shifted (Fsd), which controls AP patterning through regulating the Bcd gradient profile, Dmpd achieves its patterning role through regulating the activating potency of Bcd.

\footnotetext{
${ }^{1}$ Division of Biomedical Informatics, Cincinnati Children's Research Foundation, 3333 Burnet Avenue, Cincinnati, Ohio 45229, USA. 2 Division of Developmental Biology, Cincinnati Children's Research Foundation, 3333 Burnet Avenue, Cincinnati, Ohio, USA. Correspondence and requests for materials should be addressed to J.M. (email: jun.ma@cchmc.org).
} 
E mbryonic patterning along the anterior-posterior (AP) axis of the Drosophila embryo is initiated by the action of the morphogenetic protein Bicoid $(\mathrm{Bcd})^{1,2}$. Bcd is a transcriptional activator that stimulates the expression of APpatterning genes ${ }^{3,4}$. One such gene is hunchback $(h b)$, which, in response to the Bcd concentration gradient, has an expression domain in the anterior half of the embryo ${ }^{5,6}$. It is well documented that, based on morphological and molecular markers, altering the $b c d$ gene dose maternally can shift the fatemap landscape in the embryo ${ }^{7,8}$. Experimental perturbations of the Bcd gradient properties such as the profile shape and precision can cause corresponding changes in the expression boundary of $h b$, further supporting a threshold-dependent action of Bcd in transcriptional activation during development ${ }^{3,4,7,9-11}$. Aside from its concentration-dependent action, there is another property of Bcd that is inherent to a transcriptional activator, namely, its ability (or potency) to activate target gene transcription. There have been studies investigating the activation domains of $\mathrm{Bcd}$ and the regulation of its activity ${ }^{12-17}$. However, it remains unknown-owing in part to a lack of adequate quantitative tools in the earlier studies-how the activating potency of Bcd may specifically have an impact on the AP-patterning network at a mechanistic level.

F-box proteins play important regulatory roles in many molecular and cellular processes ${ }^{18-22}$. These proteins often exist as subunits of the SCF (Skp1-Cul1/Cdc53-F-box protein)-type ubiquitin E3 ligases, representing the specificity determinants of these protein complexes ${ }^{22-24}$. A well-documented function of F-box proteins is their ability to bind specifically to protein substrates and target them for ubiquitination and proteasomemediated degradation. In a recent study, we reported the identification of a novel F-box protein, Fate-shifted (Fsd), that has a role in targeting Bcd for ubiquitination and degradation ${ }^{10}$. Embryos mutant for $f s d$ have an altered (elongated) Bcd gradient profile and, as a result, a posterior shift in $h b$ expression boundary and the entire fatemap. These results provide a fresh example illustrating the important roles of F-box proteins in regulating developmental fate specification.

In addition to the regulation of substrate stability, F-box proteins have also emerged to have an ability to stimulate the activity of transcription factors ${ }^{25-27}$. For example, the F-box protein Skp2 can act as a co-activator for c-Myc-induced transcription in addition to regulating c-Myc stability ${ }^{28,29}$. Another F-box protein, $\beta$-TrCpl/Fbwla, has been shown to enhance the ability of $\beta$-catenin to activate transcription through cooperating with the co-activator p300 in addition to targeting $\beta$-catenin for degradation ${ }^{30}$. We were interested in understanding whether the activating potency of $\mathrm{Bcd}$ might be subject to regulation by F-box proteins. A major motivation of our interest in this question lied at the promise that the Bcd gradient system should be suitable for dissecting the mechanistic details of transcriptional regulation in a native biological system with fine resolutions both temporally and spatially. Our analysis of Fsd indicated that this F-box protein does not regulate the activating potency of Bcd (see below for details), which is consistent with the fact that Fsd is predominantly localized to the cytoplasm ${ }^{10}$.

Here we present studies of another novel F-box protein, encoded by dampened ( $d m p d$, CG11866), which is a nuclear cofactor of Bcd. We show that embryos from female mutants for $d m p d$ have a decreased $h b$ mRNA level at nuclear cycle (nc) 14 , a defect that can be traced back to a reduction in $h b$-transcribing probability (hence, the gene name dampened). The lowered amplitude (level) of $h b$ expression can be propagated into the APpatterning network to alter the spacing of even-skipped (eve) stripes that are sensitive to absolute concentrations of $\mathrm{Hb}$. These results demonstrate that the activating potency of Bcd is subject to regulation for the embryo to achieve a normal AP-patterning outcome. The contrasting properties of Fsd and Dmpd also illustrate that two distinct properties of Bcd as a morphogen-its concentration gradient profile and its ability to activate transcription-are subjected to regulation by distinct F-box proteins.

\section{Results}

Nuclear F-box protein Dmpd regulates Bcd activity. In our efforts to study the roles of F-box proteins in regulating $\mathrm{Bcd}$ functions, we identified Dmpd as a nuclear cofactor of Bcd. Figure 1a,b shows the results of a co-immunoprecipitation (co-IP) assay, where we co-transfected the human embryonic kidney (HEK) $293 \mathrm{~T}$ cells with plasmids expressing haemagglutinin (HA)-tagged Bcd (HA-Bcd) and FLAG-tagged Dmpd (FLAGDmpd). HEK293T cells are widely used as a host system to study F-box protein functions ${ }^{10,31}$. Here we used anti-HA antibody to pull down HA-Bcd in co-IP and anti-FLAG antibody to detect FLAG-Dmpd in western blots (see Fig. 1a,b, lanes 5 and 6, for control experiments documenting antibody specificity). Figure 1a shows the western blot results where FLAG-Dmpd was specifically co-precipitated by HA-Bcd (lane 4 ; see other lanes for controls). In a reciprocal setting, where anti-FLAG antibody was used to pull down FLAG-Dmpd in co-IP and anti-HA antibody was used to detect HA-Bcd in western blots, we obtained equivalent results (Fig. 1b, lane 4, see other lanes for controls), further documenting an interaction between HA-Bcd and FLAG-Dmpd.

Immunocytochemistry in Drosophila S2 cells reveals that Dmpd is almost exclusively localized to the nucleus (Fig. 1d-f), suggesting that it may play a role in regulating the function of Bcd as a transcriptional activator. To test this possibility, we used a reporter gene encoding chloramphenicol acetyltransferase (CAT) under the control of the $\sim 250$-bp Bcd-responsive native $h b$ enhancer element ${ }^{13,32,33}$. Figure 1c shows that the CAT reporter activity was increased $\sim 1.7$-fold by the expression of Dmpd in cells. To determine whether the action of Dmpd on reporter activation might be through a general mechanism that is independent of Bcd, we performed reporter assays where the reporter expression is activated by an artificial transcription activator Gal4-VP16 (ref. 34). Our results (Fig. 1c) show that the reporter activity in this case was not increased by Dmpd expression in cells, excluding the possibility that Dmpd stimulates transcription through a general mechanism that is independent of Bcd.

Evaluating $\boldsymbol{h} \boldsymbol{b}$ expression boundary and amplitude. To evaluate the impact of Dmpd on Bcd-activated transcription in a native developmental context, we first sought to establish a quantitative platform suitable for simultaneously but specifically evaluating the two parameters of a response to the Bcd input: the amplitude and boundary position of $h b$ expression in the embryo. While previous studies in Drosophila embryos have focused extensively on the regulation of $h b$ expression boundary ${ }^{11,35-40}$, relatively little attention has been paid to the regulation of the amplitude of Bcd target gene expression. We chose $h b$ for evaluating the regulation of Bcd-activating potency because, unlike most APpatterning genes that also respond to other inputs ${ }^{41,42}, h b$ responds primarily to the $\mathrm{Bcd}$ input ${ }^{9,43}$, which should make it a sensitive target suitable for such evaluations. Gap genes such as $h b$ are located at the top of the hierarchy of the regulatory cascade of the AP-patterning network to establish the earliest interpretations of the maternal inputs. For our experiments, we used quantitative fluorescence in situ hybridization (FISH) to detect mature $h b$ mRNA in the cytoplasm of the blastoderm 
a
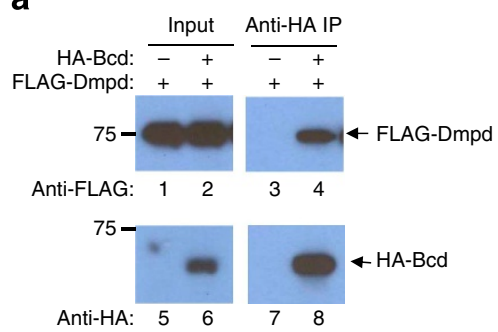

d

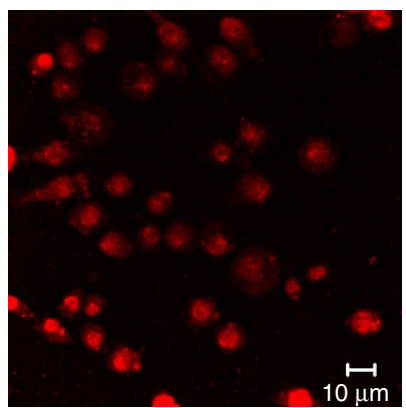

b

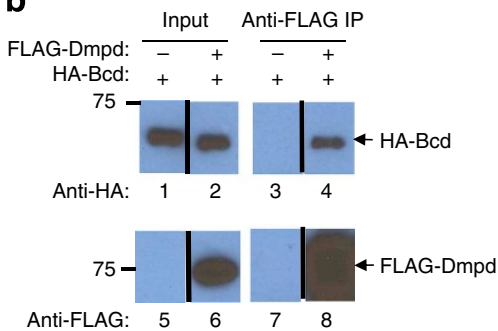

e

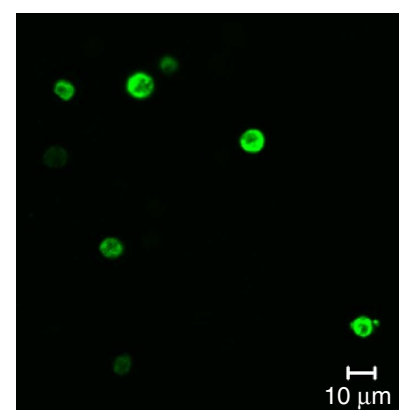

C

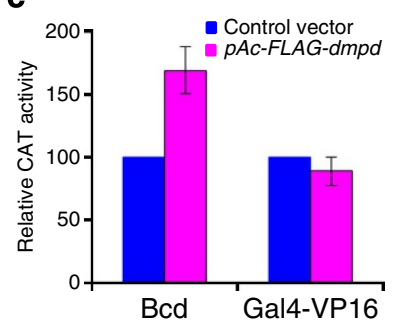

$\mathbf{f}$

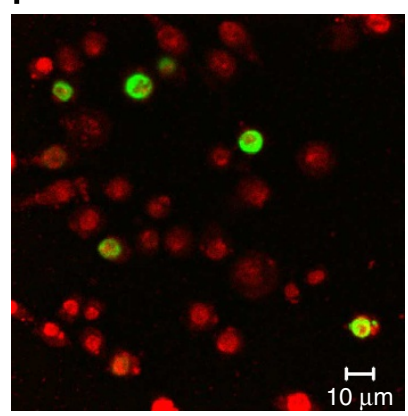

Figure 1 | Dmpd is a nuclear factor that stimulates Bcd-dependent reporter activity in cells. (a,b) FLAG-Dmpd interacts with HA-Bcd. co-IP experiments were performed to detect the interaction between FLAG-Dmpd and HA-Bcd. Here extracts from HEK293T cells expressing the indicated fusion proteins were subjected to co-IP by the indicated antibodies, followed by western blot detections by the indicated antibodies. Lanes 5 and 6 in these panels document the specificity of the antibodies used in our study. (c) Bcd activity is stimulated by Dmpd. Reporter assays were performed in Drosophila S2 cells to evaluate the impact of Dmpd expression on CAT reporter activity. For each set of experiments, CAT activities were normalized to those obtained from cells without Dmpd expression (set as 100). Data shown are from three independent sets of experiments, with s.d. shown for Dmpd-expressing cells. (d-f) Nuclear localization of Dmpd. A plasmid expressing the HA-tagged Dmpd protein (HA-Dmpd) was transfected into S2 cells. Immunocytochemistry experiments were then performed to evaluate its subcellular localization through the use of the anti-HA antibody (e). To-pro-3 was used to counterstain the nucleus (d). Panel $\mathbf{f}$ is a merge of images shown in panels $\mathbf{d}$, e. Scale bar is $10 \mu \mathrm{m}$.

embryo. To maintain a linear relationship between the fluorescence signal intensity and $h b$ mRNA level, we avoided any nonlinear signal amplification steps and ensured that all images were captured within a linear range. In addition, for any comparisons to be made, all the experimental and imaging steps were performed on a side-by-side basis. Under these conditions, the un-normalized, raw fluorescent intensities should allow us to compare directly the $h b$ mRNA levels among different groups of embryos and, as detailed below, we used calibrating embryos to specifically validate this point. To process the captured images, we first extracted the FISH intensities at locations within the basal cytoplasmic layer along the dorsal side of an embryo. The extracted intensity data were then projected to the AP axis and expressed as a function of fractional embryo length (EL) $x / L$. For our study, we defined the $h b$ expression boundary, $x_{\mathrm{hb}}$, as the AP position at which the detected FISH intensity is at half maximal. We quantify the amplitude of Bcd-activated $h b$ expression through the use of $h b_{\text {plat }}$, which denotes the averaged un-normalized, raw intensity at positions in the plateau region of the anterior $h b$ expression domain of an embryo $^{44}$.

We used calibrating embryos to test and validate our quantitative platform outlined above. These embryos have one, two or four copies of the $h b$ gene (referred to as $1 \times, 2 \times$ and $4 \times h b$ embryos, respectively). Here side-by-side quantitative FISH experiments were performed in these embryos to detect and quantify mature $h b$ mRNA. To ensure reliable comparison of $h b$ expression properties, embryos were specifically selected to be at a time ( $\sim 5-15 \mathrm{~min}$ into nc 14$)$ when $h b_{\text {plat }}$ is known to have reached its stable, peak levels ${ }^{44}$. The only adjustment in our analysis was an embryo-specific background subtraction step that eliminated embryo-to-embryo differences in terms of background fluorescence. Figure $2 \mathrm{a}$ shows the mean $h b$ intensity profiles from $1 \times, 2 \times$ and $4 \times h b$ calibrating embryos (see Supplementary Fig. S1. for individual intensity profiles). These profiles exhibit a classical and well-documented sigmoidal shape, each with a sharp boundary. Figure $2 b$ shows that the mean $h b_{\text {plat }}$ values exhibit a linear relationship with the $h b$ copy number $\left(h b_{\text {plat }}=5.00 \times c+0.86\right.$, where $c$ is $h b$ copy number; $R$ squared for linear regression $\left.R^{2}=0.75\right)$. These results show that $h b_{\text {plat }}$ detected under our experimental and analytical framework is a sensitive and reliable measure of the mature $h b$ mRNA level and thus a valid parameter for quantifying the amplitude of a Bcd target gene response in the embryo.

Since $h b$ expression is dependent on Bcd function, we sought to further document in an unambiguous manner whether $h b_{\text {plat }}$ is, or is not, sensitive to changes in $b c d$ gene copies. Here our goal was to document specifically whether $h b_{\text {plat }}$ is sensitive to the $h b$ boundary position or not. We performed quantitative FISH to detect $h b$ mRNA in embryos from females that have one, two or three copies of the $b c d$ gene (referred to as $1 \times, 2 \times$ and $3 \times b c d$ embryos, respectively; see Supplementary Fig. S2 for individual intensity profiles). Figure $2 \mathrm{c}, \mathrm{d}$ show our quantitative data. They confirm that $x_{\mathrm{hb}}$ is a function of bcd gene copy number, exhibiting a progressive posterior shift as the bcd gene copy number increases. However, the mean $h b_{\text {plat }}$ values remain similar in $1 \times, 2 \times$ and $3 \times b c d$ embryos (Fig. $2 \mathrm{~d}$ ). These results further document that $h b_{\text {plat }}$ quantifies exclusively how much $h b$ mRNA is produced irrespective of where its expression boundary is located in the embryo. They provide further confirmation that $h b_{\text {plat }}$ is a valid and specific parameter for quantifying the amplitude of a Bcd target gene response. 
a

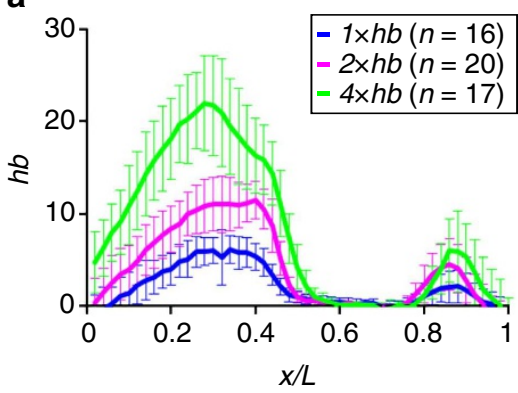

C

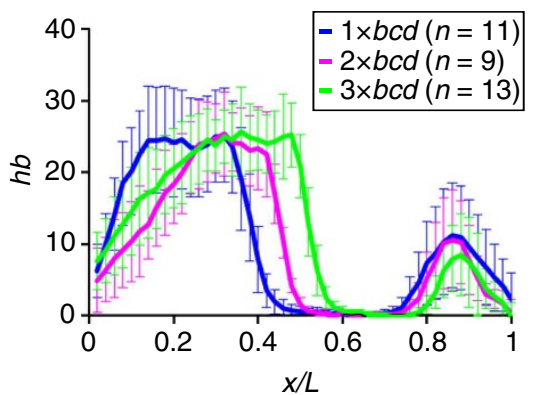

b

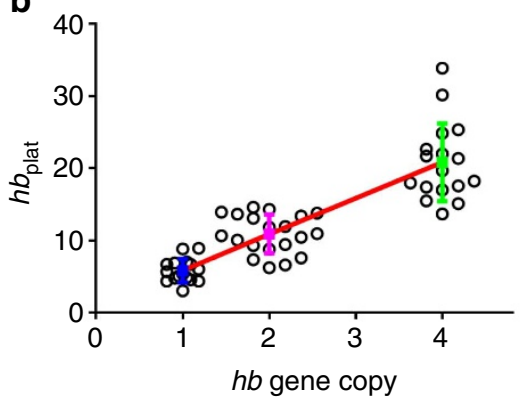

d

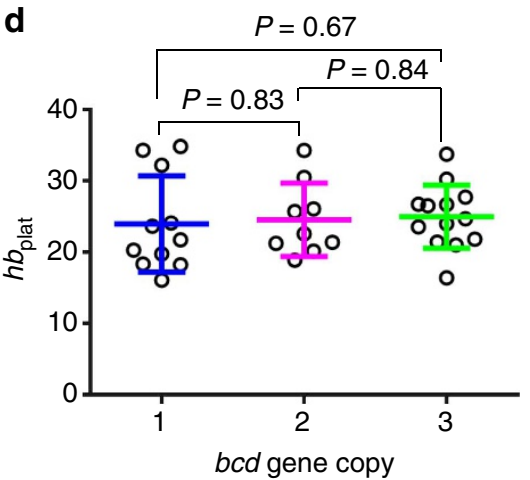

Figure 2 | Establishing a platform for quantifying the amplitude of $\boldsymbol{h} \boldsymbol{b}$ transcription. (a) Shown are the mean profiles (with s.d. shown) of un-normalized $h b$ mRNA FISH intensities (in a.u.'s) from embryos that have one, two or four copies of the $h b$ gene. The mean and s.d. of $x_{h b}$ expressed as fractional embryo length are: $0.435 \pm 0.013,0.445 \pm 0.013$ and $0.454 \pm 0.019$ for $1 \times, 2 \times$ and $4 \times h b$ embryos, respectively ( $n=16,20$ and 17 ). These results show that $x_{\mathrm{hb}}$ remains largely insensitive to changes in $h b$ copy number in our experimental system; a relatively minor shift might be related to the limitations inherent to experimental detections ${ }^{35}$. (b) Shown are $h b_{\text {plat }}$ values (in a.u.'s) in individual embryos with different copies of the $h b$ gene. The mean and s.d. are also shown in the figure $\left(n=16,20\right.$ and 17 for $1 \times, 2 \times$ and $4 \times h b$ embryos, respectively). The linear fit between $h b_{\text {plat }}$ and $h b$ gene copy number is shown as a red line in the figure. (c) Shown are the mean profiles of un-normalized $h b$ mRNA FISH intensities in embryos from mothers with one, two or three copies of $b c d$. The mean and s.d. of $x_{\mathrm{hb}}$ expressed as fractional EL are: $0.373 \pm 0.013,0.446 \pm 0.009$ and $0.510 \pm 0.010$ for $1 \times, 2 \times$ and $3 \times b c d$ embryos, respectively $\left(n=11,9\right.$ and 13). (d) Shown are $h b_{\text {plat }}$ values in individual embryos from mothers with different copies of bcd. The mean and s.d. of $h b_{\text {plat }}$ are: $23.93 \pm 6.75,24.51 \pm 5.13$ and $24.94 \pm 4.42$ for $1 \times, 2 \times$ and $3 \times$ bcd embryos, respectively $(n=11,9$ and 13$)$. $P$-values (obtained from Student's $t$-tests and shown in the figure) reveal no significant differences.

dmpd mutation reduces $h b$ mRNA level in the embryo. With the established quantitative platform outlined above, we proceeded to investigate the potential role of Dmpd in regulating the activating potency of Bcd during development. For this purpose, we generated a deletion allele $d m p d^{6-3}$ through P-elementmediated imprecise excision using a publicly available insertion line (Bloomington fly stock 17518 ). The resulting $d m p d^{6-3}$ allele has a 3,106-bp deletion covering almost the entire coding region of $d m p d$ (Fig. 3a). It is homozygous viable at $25^{\circ} \mathrm{C}$, which made it possible for us to directly collect embryos from homozygous $d m p d^{6-3}$ females. Using our quantitative tools described above, we evaluated specifically the effect of the dmpd mutation on $h b$ mRNA profiles in the embryo. Here we performed quantitative FISH in embryos from $w^{1118}$ or $d m p d^{6-3}$ mothers, referred to as wt and $d m p d$ embryos, respectively. Figure $3 \mathrm{~b}$ shows the mean profiles of $h b$ mRNA FISH intensities (raw, background-subtracted) from wt and $d m p d$ embryos (see Supplementary Fig. S3 for individual profiles), revealing a lower intensity level in $d m p d$ embryos. Figure $3 \mathrm{c}$ shows that $h b_{\text {plat }}=30.98 \pm 6.18$ (all values given in this report are mean \pm s.d. unless stated otherwise; $n=22)$ and $18.10 \pm 5.10 \quad(n=18)$ in wt and dmpd embryos, respectively $\left(P=2.04 \times 10^{-8}\right.$; all $P$-values given in this report are from Student's $t$-tests unless stated otherwise), documenting a significant reduction in Bcd-activated $h b$ expression amplitude in $d m p d$ embryos. The observed defect of $d m p d$ embryos in $h b$ amplitude reduction is Bcd-dependent because the amplitude of the posterior $h b$ expression domain, which is driven by a Bcdindependent enhancer ${ }^{45}$, was unaffected $\left(h b_{\text {post }}=9.99 \pm 5.77\right.$ and $10.14 \pm 4.36$ in wt and $d m p d$ embryos, respectively; $P=0.93$; see Fig. $3 c$ for $h b_{\text {post }}$ definition). In addition, the embryos chosen in our evaluation of $h b$ mRNA levels are at early nc 14 since the $h b$ expression stripe at parasegment 4 (PS4), which is dependent on gap gene cross-regulatory inputs ${ }^{36,45}$, has not yet gained any appreciable prominence (Fig. 3b). Together, these findings suggest that the activating potency of Bcd is subject to positive regulation by Dmpd during development.

To evaluate the specificity of our $d m p d$ deletion allele, we performed the following two sets of experiments. First, we compared $h b_{\text {plat }}$ between wt embryos and those from $d m p d^{6-3} /$ $D f(2 R) B S C 303$ females (referred to as $d m p d / D f$ embryos). $D f(2 R) B S C 303$ is a small deficiency $(\sim 70 \mathrm{~kb})$ that removes the entire $d m p d$-coding region. Our results (Supplementary Fig. S4) show that, similar to $d m p d$ embryos, $d m p d / D f$ embryos also have a reduced $h b_{\text {plat }}$ from $25.05 \pm 5.47(n=10)$ in wt embryos to $17.93 \pm 4.03(n=12)$ in $d m p d / D f$ embryos $\left(P=2.20 \times 10^{-3}\right)$. Second, we backcrossed $d m p d^{6-3}$ females with $w^{1118}$ males for six generations to establish a $d m p d^{6-3}$ line in $w^{1118}$ background, referred to as $d m p d^{6-3 w}$. We performed quantitative $h b$ FISH in embryos from $w^{1118}$ or $d m p d^{6-3 w}$ females, further confirming a reduction in $h b_{\text {plat }}$ in $d m p d^{6-3 w}$ embryos (Supplementary Fig. S5). In both sets of experiments, $h b_{\text {post }}$ was unaffected (Supplementary Figs S4f,S5f). Together, these results document that the observed 
a

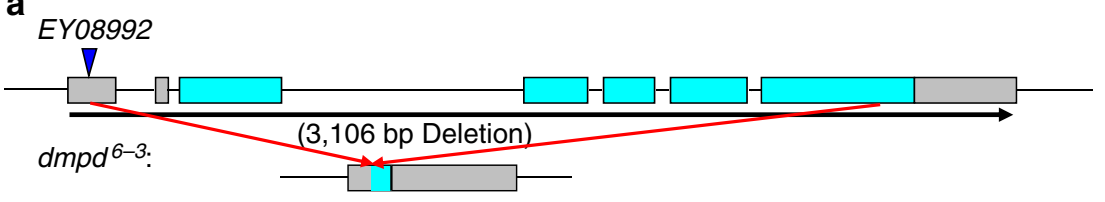

b

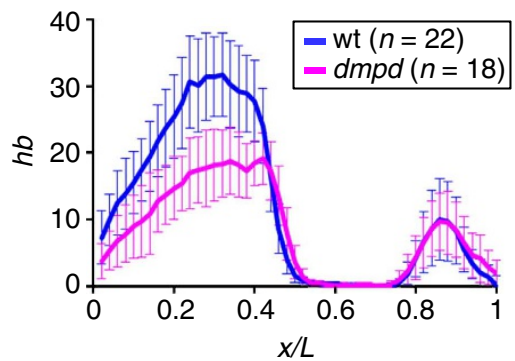

C

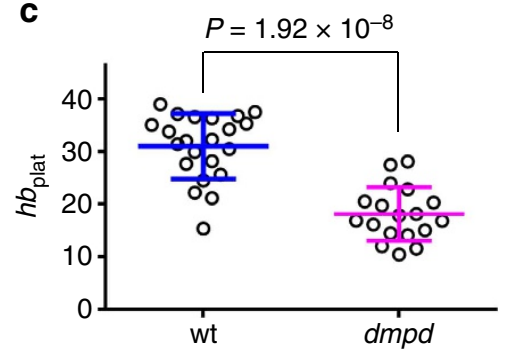

Figure 3 | The $\boldsymbol{h} \boldsymbol{b}$ mRNA level is reduced in dmpd embryos. (a) A schematic diagram showing the deletion caused by the $d^{\text {mpd }} d^{6-3}$ allele. The boxes represent exons with the annotated open reading frame in cyan and the untranslated regions in grey. The blue arrow shows the P-element insertion site for

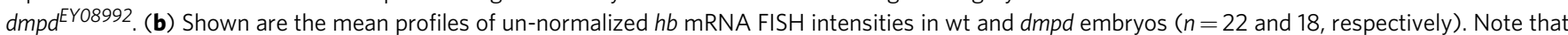
the level of the Bcd-independent posterior $h b$ expression domain remains unaffected in dmpd embryos ( $P=0.93$, Student's $t$-test); see text and $\mathbf{c}$ legend for further details). The mean and s.d. of $x_{\mathrm{hb}}$ expressed as fractional embryo length are: $0.433 \pm 0.012$ and $0.462 \pm 0.018$ for wt and $d m p d$ embryos, respectively $\left(P=4.8 \times 10^{-7}\right.$, Student's $t$-test). (c) Shown are $h b_{\text {plat }}$ values in individual wt and $d m p d$ embryos $(n=22$ and 18 , respectively). Unlike the significant amplitude reduction for the Bcd-activated anterior $h b$ expression domain shown in this figure, the amplitude of the Bcd-independent posterior $h b$ expression domain, $h b_{\text {post }}$ (defined as the average FISH intensities at the three peak positions of the posterior domain), is insensitive to dmpd mutation.

$h b_{\text {plat }}$ reduction is a defect that is caused specifically by the $d m p d$ mutation (as opposed to genetic background differences). They further support a role of Dmpd in stimulating specifically Bcddependent $h b$ transcription in the embryo.

dmpd mutation alters AP-patterning characteristics. $\mathrm{Hb}$ regulates AP patterning by controlling the expression boundaries of downstream genes in a concentration-dependent manner ${ }^{16,17,46}$. Specifically, the spacing between the anterior boundaries of eve stripes 3 and 4 (referred to as $\Delta E L_{\text {eve3-4) }}$ ) is known to be controlled by the absolute $\mathrm{Hb}$ concentrations in the embryo ${ }^{47,48}$. A reduction in the absolute $\mathrm{Hb}$ level (in $h b /+$ heterozygous embryos) has a well-documented consequence of enlarging $\Delta E L_{\text {eve3-4 }}{ }^{47,48}$. To determine whether the effect of Dmpd on $h b$ transcription (that is, the reduced amplitude) can be propagated further downstream of the AP-patterning network, we performed quantitative FISH to detect eve expression patterns in wt and $d m p d$ embryos for a direct comparison of $\Delta E L_{\text {eve3-4. }}$. Figure $4 \mathrm{a}$ shows the mean intensity profiles of eve in wt and $d m p d$ embryos (see Supplementary Fig. S6 for individual profiles). Figure 4b shows the measured $\Delta E L_{\text {eve3-4 }}$ in these embryos, documenting a significantly larger $\Delta E L_{\text {eve3-4 }}$ in $d m p d$ embryos $(7.60 \pm 0.37 \% \mathrm{EL}$, $n=13)$ than in wt embryos $(6.34 \pm 0.45 \%$ EL, $n=16$; $\left.P=9.26 \times 10^{-9}\right)$. These results show that the impact of the reduced amplitude of $h b$ expression caused by the $d m p d$ mutation can be faithfully propagated downstream of the AP-patterning network, indicating a role of Dmpd in specifying a normal APpatterning outcome.

$\boldsymbol{d m p d}$ reduces $\boldsymbol{h} \boldsymbol{b}$-transcribing probability at nc 14 . Our study described thus far shows that Dmpd can enhance the activating potency of Bcd and is required for a normal AP-patterning outcome. To investigate at a deeper mechanistic level how Bcdactivated $h b$ transcription becomes compromised in $d m p d$ embryos, we employed a technique that we developed recently for monitoring active $h b$ transcription ${ }^{44}$. Here we used an intronic probe to detect the nascent $h b$ transcripts near the Bcd-responsive P2 promoter (see Fig. 5a,b for images of a wt embryo at nc 14). Since the 283-bp $h b$ intron is very close to the P2 promoter (145 bp downstream) and since RNA intron sequences are believed to be quickly removed upon being transcribed ${ }^{49}$, our technique permits a close monitoring of the transcriptional status of individual $h b$ gene copies near the Bcd-responsive promoter. In our analysis, the fluorescence dots detected inside the nucleus (referred to as the intron dots) represent individual copies of $h b$ undergoing active transcription in snapshots of the embryos. For each embryo, we first calculated the mean intron dot number per nucleus $(\rho)$ at positions along the AP axis (Fig. 5c). This parameter quantifies the probability of $h b$ gene copies undergoing active transcription (referred to as transcribing probability) at an AP position in a snapshot of the embryo. Specifically, $\rho=2$ indicates that all $h b$ copies in the nuclei at the AP position under investigation are actively transcribing in the snapshot, thus denoting a transcribing probability of $100 \%$. To specifically evaluate Bcd-activated $h b$ transcription, we also calculated the average $\rho$ for positions in the plateau region of the $h b$ expression domain, $\rho_{\text {plat }} 4$. To minimize the effects of both PS4 and the variable anterior stripe ${ }^{6,45}$ on the calculation of $\rho_{\text {plat }}$, the plateau region was chosen to be a domain that spans $10 \% \mathrm{EL}$ and has a posterior boundary that is $\sim 10 \% \mathrm{EL}$ anterior to the detected $h b$ expression boundary of the embryo. The parameter $\rho_{\text {plat }}$ thus provides a quantification of the maximal Bcd-dependent transcribing probability of $h b$ gene copies in a snapshot of the embryo.

To obtain an accurate comparison of $h b$-transcribing probability between wt and $d m p d$ embryos, we grouped embryos into well-resolved temporal classes. These time classes have a temporal resolution of $\sim 1 \mathrm{~min}$ with an uncertainty of $\sim 0.6$ to $\sim 0.9 \mathrm{~min}$ (ref. 44). Figure $5 \mathrm{~d}-\mathrm{i}$ shows the mean $\rho$ profiles in wt and $d m p d$ embryos at time classes from t1 to t6 (see Supplementary Figs S7,S8 for individual profiles). These profiles show a reduced $\rho$ level at time classes $\mathrm{t} 3-\mathrm{t} 6$, which represent embryos that are $\sim 3$ to $\sim 6 \mathrm{~min}$ into nc 14 interphase (see Discussion for $\rho$ properties 
a

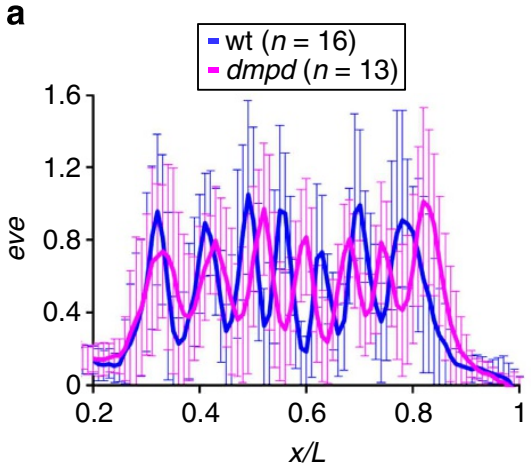

b

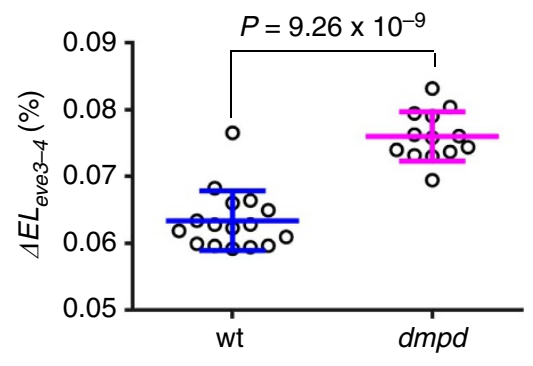

Figure 4 | The spacing between eve stripes 3 and 4 is sensitive to the dmpd mutation. (a) Shown are the normalized mean intensity profiles of eve in wt and dmpd embryos ( $n=16$ and 13, respectively). (b) Shown is the calculated distance between eve stripes 3 and 4 in each individual wt and dmpd embryos ( $n=16$ and 13, respectively). The distance values shown are expressed as percentage of embryo length. Also shown are the mean and s.d. for these two groups, along with the $P$-value obtained from Student's $t$-test.

a
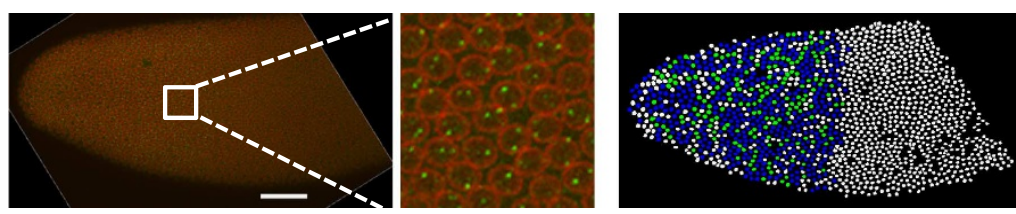

d

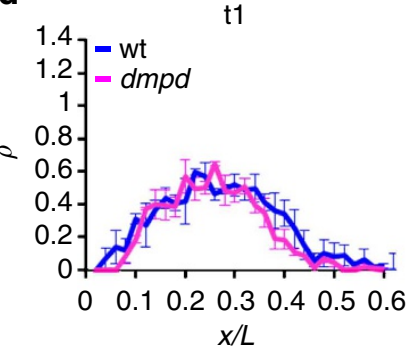

g

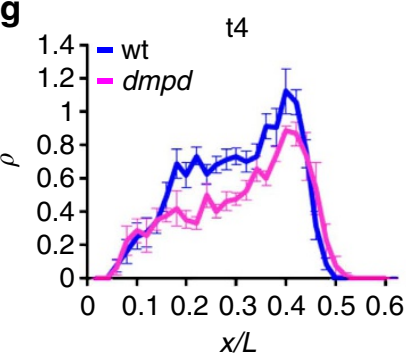

b

e

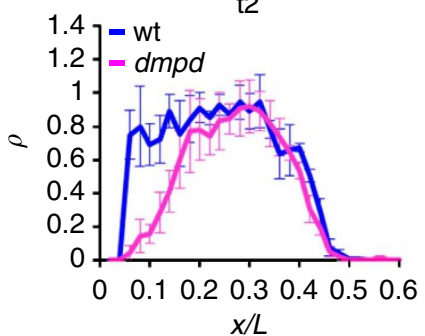

h

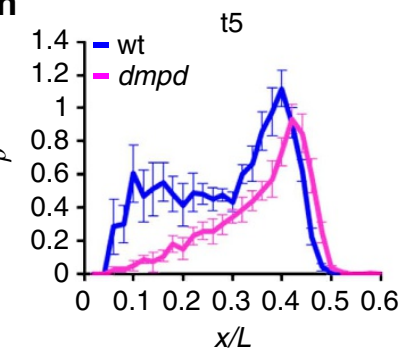

C

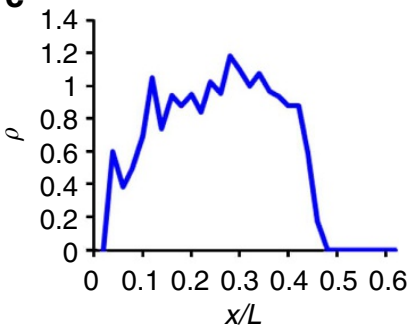

f

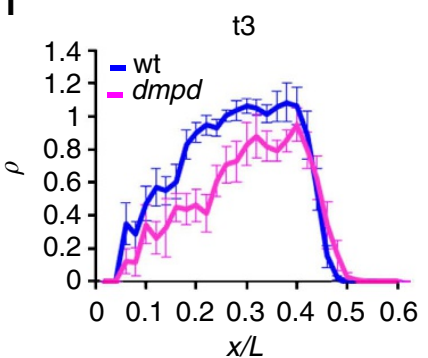

i

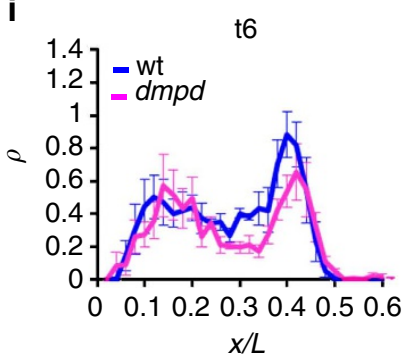

Figure 5 | The transcribing probability near Bcd-responsive promoter of $\boldsymbol{h} \boldsymbol{b}$ is decreased in dmpd embryos. (a) Shown is an image of $h b$ intron staining from a cycle 14 embryo. Nuclear envelope is shown in red and intron dots in green. Scale bar, $50 \mu \mathrm{m}$. A magnified view of a section of the expression domain is shown on the right side of this panel. (b) Shown is a computer-processed image of panel (a). Here each of the identified nuclei is shown in a colour according to the number of intron dots detected inside. White, blue and green represent zero, one and two intron dots detected, respectively. (c) Shown is the plot of $\rho$ as a function of $x / L$ based on panel $\mathbf{b}$. (d-i) Shown are the mean $\rho$ profiles from wt and dmpd embryos at time classes $t 1-t 6$. The mean and s.d. of $\rho_{\text {plat }}$ for t1-t6 time classes are: $0.50 \pm 0.07 ; 0.84 \pm 0.04 ; 1.03 \pm 0.08 ; 0.71 \pm 0.14 ; 0.52 \pm 0.10 ; 0.34 \pm 0.03$ for wt embryos $(n=3,3,7$, 7,5 and 4, respectively) and $0.51 \pm 0.11 ; 0.86 \pm 0.25 ; 0.79 \pm 0.21 ; 0.54 \pm 0.06 ; 0.38 \pm 0.12 ; 0.23 \pm 0.05$ for dmpd embryos $(n=3,4,5,5,5$ and 4$)$, respectively. The $P$-values (from Student's $t$-tests) between wt and dmpd embryos for t1-t6 time classes are: 0.93; 0.92; 0.016; 0.028; 0.078; 0.0087, respectively. The mean $\rho$ at $x / L \sim 0.15$ (which is located outside of the plateau domain for calculating $\rho_{\text {plat }}$ ) appears higher in dmpd embryos than in wt embryos at $t 6$ (i); this is likely caused by an 'outlier' dmpd embryo that has a very high level of $\rho$ at this location (see Supplementary Fig. S8f). 
at $\mathrm{t} 1$ and $\mathrm{t} 2$ ). For example, the peak transcribing probability is reached in wt embryos at $\mathrm{t} 3$ with a mean $\rho_{\text {plat }}$ of $1.03 \pm 0.08$ $(n=7)$. By contrast, $d m p d$ embryos at this time class have a significantly lower transcribing probability with a mean $\rho_{\text {plat }}$ of $0.79 \pm 0.21\left(n=5 ; P=1.6 \times 10^{-2}\right.$; see Fig. 5 legend for $P$-values for other time classes). These results uncover an underpinning defect caused by the $d m p d$ mutation, namely, a reduction in the transcribing probability of individual copies of the $h b$ gene in the embryo. Importantly, the estimated contributions of active $h b$ transcription at PS4 are insensitive to $d m p d$ mutation in embryos that have developed a detectable PS4 stripe in the form of intron dots (see Supplementary Fig. S9 and legend). It is worth mentioning that, while the roles of cofactors in modulating the transcriptional outcome are well documented ${ }^{50}$, our finding that Dmpd regulates the transcribing probability of a native Bcd target gene in a native developmental system represents a significant advancement in our understanding of mechanisms of transcriptional regulation.

dmpd does not affect Bcd gradient profile. F-box proteins can enhance activator function through a variety of mechanisms ${ }^{27,51-53}$. They can be either proteolytic or non-proteolytic in nature as defined by their ability to regulate activator stability. To investigate whether Dmpd has a role in regulating $\mathrm{Bcd}$ degradation in addition to its co-activator function, we evaluated the Bcd gradient properties in $d m p d$ embryos. Here we performed quantitative immunostaining to detect the Bcd protein in wt and dmpd embryos ${ }^{10,11}$. We extracted raw Bcd intensity without any adjustments, $B$, at different positions along the AP axis for individual embryos (see Supplementary Fig. S10 for the mean and individual profiles of $\mathrm{Bcd}$ intensities). According to a simple diffusion model, the length constant of an exponential Bcd gradient profile, $\lambda$, is a function of the diffusion constant $D$ and degradation rate $\omega: \lambda^{2}=D / \omega$ (refs 38,54 ). In $d m p d$ embryos, $D$ is expected to be unchanged since Bcd itself is not altered, thus making it possible to use the estimated parameter $\lambda$ to evaluate a potential impact of $d m p d$ mutation on $\omega$. Figure 6 a shows $\ln \left(B / B_{\max }\right)$ plots for the mean Bcd intensity profiles from wt and $d m p d$ embryos (normalized to their respective mean maximal $B$ value, $B_{\max }$ ) as a function of $x / L$ (see Fig. 6 legend for further details). Here the logarithmic conversion transforms an exponential profile to a linear profile, where $\lambda$ is the negative reciprocal of the slope. Our results show that the two linear fits have a similar slope, suggesting a similar $\lambda$ between wt and $d m p d$ embryos. In a second analysis, we calculated $\lambda$ values of $\mathrm{Bcd}$ gradient profiles from individual embryos (see Methods for

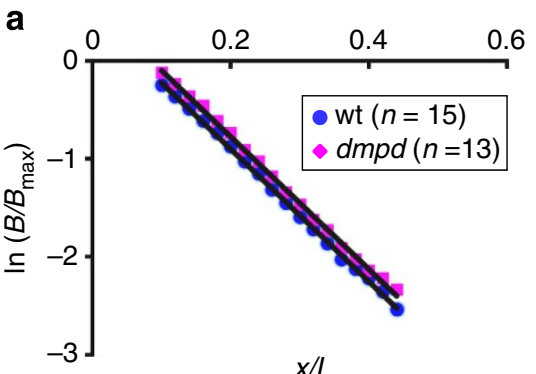

details). Our results further confirmed the similarity of the length constant, $\lambda=72.96 \pm 11.52 \mu \mathrm{m}(n=15)$ and $74.14 \pm 12.56 \mu \mathrm{m}$ $(n=13)$ in wt and $d m p d$ embryos, respectively $(P=0.80$; Fig. $6 \mathrm{~b})$. Together, these results suggest that, under the framework of a simple diffusion model, Dmpd does not have a significant role in regulating Bcd stability in embryos. They are supportive of a nonproteolytic role of Dmpd in enhancing the activating potency of Bcd.

\section{Discussion}

Morphogen gradients such as those of Bcd are excellent experimental paradigms for dissecting the mechanistic operations of the regulatory networks that control patterning decisions. They provide a unique window to probing the regulatory impacts of F-box proteins both spatially and temporally at a fine resolution. Increasing evidence supports the notion that F-box proteinsthere are up to 45 of them in Drosophila and 75 in humans ${ }^{55}$ belong to a critical class of regulatory proteins; however, few of them have been studied in native developmental contexts. In this work, we report the identification of a nuclear cofactor of $\mathrm{Bcd}$, Dmpd, that can enhance the potency of Bcd as an activator. Dmpd thus joins an expanding list of F-box proteins that can act as transcriptional co-activators ${ }^{28-30}$; it remains to be determined whether the co-activator role of Dmpd for Bcd in the embryo is dependent on a functional SCF complex and the E3 ligase activity. Our results show that embryos lacking Dmpd have a lower amplitude of Bcd-activated $h b$ expression, a defect that can be passed further downstream of the network, causing an enlargement of $\Delta E L_{\text {eve3-4, }}$, the spacing between eve expression stripes that are sensitive to absolute concentrations of $\mathrm{Hb}$. In embryos that lack another Bcd-interacting F-box protein Fsd, neither the $h b$ expression amplitude nor $\Delta E L_{\text {eve3-4 }}$ is affected (Supplementary Fig. S11). This stems from the fact that, unlike Dmpd, Fsd regulates the Bcd gradient profile through a proteolytic pathway without a detectable co-activator function (Liu and $\mathrm{Ma}^{10}$ and Supplementary Fig. S11). The contrasting functions of these two F-box proteins thus document that a normal AP-patterning outcome is subject to regulation by two distinct mechanisms. These mechanisms control two distinct properties of Bcd that are indispensable to its morphogen action: the formation of a concentration gradient and the activation of its target genes.

A critical feature of morphogen gradients is their ability to induce downstream responses in a concentration-dependent manner. This particular feature has been subjected to extensive investigations, in part because of a significant interest in the question of what morphogen gradients do. It has been proposed

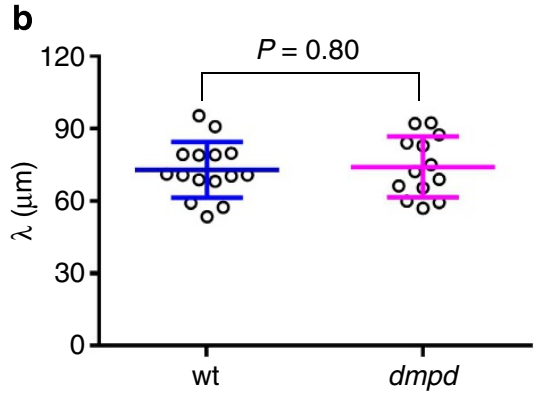

Figure 6 | Similar length constant of the Bcd gradient profiles in wt and dmpd embryos. (a) Shown are the mean Bcd gradient profiles from wt and dmpd embryos plotted as $\ln \left(B / B_{\max }\right)$ against $A P$ position $x / L$. Here both $B$ and $B_{\max }$ are background-adjusted intensities without any further adjustments. The solid lines represent linear fits $\left(y=-6.78 x+0.46, R\right.$ squared for linear regression; $R^{2}=0.999$ for wt embryos; $y=-6.77 x+0.58 ; R$ squared for linear regression, $R^{2}=0.998$ for dmpd embryos). (b) Shown are calculated $\lambda$ values of Bcd gradient profiles from individual wt and $d m p d$ embryos ( $n=15$ and 13, respectively). These values were obtained from fitting the individual Bcd intensity profiles to an exponential function. Shown are the mean and s.d. for these two groups, along with the $P$-value obtained from Student's $t$-test. 
recently that the concentration-dependent input-output relationship between $\mathrm{Bcd}$ and $h b$ also contributes directly to the formation of AP patterns that are scaled with the length of the embryo $^{11,56-58}$. By contrast, the regulation of the amplitude of a response to the morphogen input has been relatively underexplored. Its importance may be better appreciated from the perspective of regulatory networks that control the patterning outcome. In the case of $h b$ as a direct target gene of Bcd, its encoded protein $\mathrm{Hb}$ acts as an input, in a concentrationdependent manner ${ }^{47,48}$, for genes (such as eve) that are further downstream of the AP-patterning network. The regulation of the expression of these downstream genes allows the regulatory network to refine and evolve towards the desired final outcome of patterning. Since these downstream genes respond to absolute concentrations of $\mathrm{Hb}$, the boundary position for $h b$ expression in response to the Bcd gradient input has become no longer directly relevant to the decision-making processes of these genes. However, as shown by our study, another feature of the $h b$ response to the Bcd gradient input, namely its expression amplitude, remains directly relevant to the continued operation of the AP-patterning network.

Our analysis of the impact of the $d m p d$ mutation on active $h b$ transcription reveals important mechanistic insights into the regulation of the transcription process in a native developmental context. As documented recently ${ }^{44}$, Bcd-activated $h b$ transcription becomes detectable immediately upon entering the nc 14 interphase; however, it is shut off within a few minutes. Our intron-staining results (Fig. $5 \mathrm{~d}, \mathrm{e}$ ) show that, at time classes $\mathrm{t} 1$ and $\mathrm{t} 2$, the $h b$-transcribing probability at the plateau region is largely unaffected by the $d m p d$ mutation (see Fig. 5 legend for $\rho_{\text {plat }}$ and $P$-values). They suggest that the onset of active $h b$ transcription upon entering the nc 14 interphase is largely insensitive to $d m p d$ mutation. The reduction in $\rho_{\text {plat }}$ at $\mathrm{t} 3$ and subsequent time classes is thus consistent with the possibility that $d m p d$ embryos might have a hastened shutdown of active $h b$ transcription at the nc 14 interphase. To test this possibility, we performed quantitative $h b$ mRNA FISH in wt and $d m p d$ embryos with an exclusive focus on nc 13. Embryos at this stage already have a significant accumulation of $h b$ mRNA suitable for quantitative measurements that are necessary for effective comparisons between wt and $d m p d$ embryos. In addition and importantly, active $h b$ transcription is known to span the entire interphases prior to nc 14 (refs 44,59). Thus, if the defect of $d m p d$ embryos is specific to $h b$ shutdown at the nc 14 interphase, $h b$ mRNA level should remain unchanged prior to this shutdown-that is, at nc 13. This prediction is supported by our results shown in Supplementary Fig. S12. An implication of these findings is that altering the $h b$ expression amplitude at, and only at, the last interphase (nc 14) prior to cellularization and gastrulation can still have an impact on the AP-patterning outcome, suggesting that this interphase represents a critical time period in making patterning decisions forward.

An important feature of developmental systems is that their desired spatial properties must be attained within the allotted periods of time when an entire system is progressing along the irreversible temporal axis. This interconnection between the spatial and temporal aspects of the developmental systems poses significant constraints on their operation. In Drosophila, the early embryo undergoes rapid cycles of nuclear division ${ }^{60}$. This poses a constraint on the transcription process itself and the decoding of maternal gradient inputs, since mitosis is known to abort transcription ${ }^{61,62}$. It has been documented that active $h b$ transcription can resume almost immediately upon entering the interphase in the blastoderm embryo ${ }^{44,59}$; however, it remains unknown precisely how this can be achieved ${ }^{61}$. For developmental systems evolving rapidly along the temporal axis such as the early Drosophila embryo, patterning decisions may need to be made before true steady states could be achieved. In a recent study ${ }^{63}$, it was shown that how quickly a gene can resume efficient transcription upon entering the nc 14 interphase can affect the amount (the amplitude) of the gene products at a later time when such products are needed for action. Thus, it was shown that a slowed onset of snail transcription led to gastrulation defects ${ }^{63}$. In the case of $d m p d$ mutation investigated in this report, while the onset of active $h b$ transcription upon entering the nc 14 interphase is unaffected, a hastened shutdown reduces the amplitude of $h b$ expression products, a defect that alters the spatial characteristics of the patterning outcome (Fig. 4). Together, these two latest examples of dynamic regulation of transcription illustrate the importance of understanding the actual transcriptional decisions in a developmental system through the prism of time, an area of research that has only begun to be explored.

Three recent studies have reported investigations of the dynamics of transcription in early embryos. Two of the studies ${ }^{64,65}$ were based on a live-imaging technique using the MS2 coat protein system ${ }^{66,67}$. For evaluating the onset of transcription upon entering an interphase, the use of this system requires an adjustment by the delay between transcription initiation and detection of fluorescent signals at the reporter locus, as dictated by the time necessary for RNA polymerase to transcribe through the MS2 stem loop repeats and for the MS2 coat protein-green fluorescent protein to bind to these RNA repeats. With this and detection limit-imposed delay adjustments, both of the live-imaging studies are consistent with a quick onset of Bcd-dependent transcription initiation upon entering an interphase as documented in our current study and previous studies ${ }^{44,59}$. The live-imaging study of Lucas et al. ${ }^{64}$ also supports a role of Bcd in directly lengthening the time period of active transcription during an interphase. At the nc 14 interphase, both the live-imaging study of Garcia et al. ${ }^{65}$ and a third study of Little et al. ${ }^{68}$ that was performed on fixed embryos support a shutdown of $h b$ transcription that we initially reported $^{44}$ and further investigated in the current study. The results of Garcia et al. ${ }^{65}$, when adjusted by the method-dictated time delays, are also broadly consistent with our estimated kinetics of $h b$ transcription onset and shutdown. Importantly, the observation ${ }^{65}$ that a reporter gene driven by the $\sim 250$-bp Bcd-responsive $h b$ enhancer element ${ }^{13,32,42}$ is shut down at nc 14 in a manner that is broadly similar to the endogenous $h b$ shutdown is consistent with our documented role of Dmpd and the activating potency of Bcd in influencing this shutdown process. Finally, we note that the dynamic properties of $h b$ shutdown described by Little et al. ${ }^{68}$ contradict with both our findings ${ }^{44}$ and those of Garcia et al. ${ }^{65}$ Unlike our experimental and analytical framework designed to evaluate specifically the dynamics of transcription initiation or elongation near the Bcd-dependent promoter ${ }^{43,44,69}$, the active $h b$ loci detected by Little et al. ${ }^{68}$ monitor the 'aggregate' transcription status along the entire $h b$ gene length detected by the probes. This makes the fluorescent signal intensities at the active $h b$ loci an insensitive quantity in studying the dynamics of $h b$ shutdown at nc 14 . In particular, the use of this quantity as transcription activity likely has contributed to the reported mismatch between $\sim 30 \%$ reduction in 'transcription activity' and approximately threefold reduction in cytoplasmic $h b$ mRNA counts from early to late embryos at nc 14. These results support a suggestion that, upon the sharp (in magnitude) and quick (in time) shutdown of $h b$ transcription at early nc 14 as documented by our method ${ }^{44}$, the lingering signals at the 'active' $h b$ loci $^{68}$ are largely unrelated to transcription initiation or elongation near the Bcd-dependent promoter. 
A contribution of our current work is the establishment of a quantitative platform for specifically (and simultaneously) analysing the amplitude and expression boundary of a target response to the Bcd gradient input. Under our current experimental framework, these two parameters are primarily subjected to regulation by two distinct mechanisms. Our results document that Dmpd has a role in enhancing the activating potency of Bcd as an activator and in regulating the AP-patterning outcome. Interestingly, we observe a detectable, although small, posterior shift in $x_{\mathrm{hb}}$ in $d m p d$ embryos (see Fig. 3 , Supplementary Figs S4,S5, and their legends), suggesting that Dmpd may have regulatory roles beyond its primary role of regulating the amplitude of $h b$ expression. This posterior shift in $x_{\mathrm{hb}}$ cannot be simply explained by the Bcd gradient profile properties because a smaller $B_{0}$ (Bcd concentration at the anterior) in $d m p d$ embryos, if biologically meaningful, would have predicted a small shift in $x_{\mathrm{hb}}$ towards the anterior. This small shift is detectable in embryos not yet exhibiting a significant sign of PS4 expression (Fig. 3), suggesting that it is related to Bcddependent $h b$ transcription. It remains to be determined mechanistically whether Dmpd may have a meaningful role in regulating the affinity of Bcd for the $h b$ enhancer during development. We anticipate that, as we develop more experimental tools and identify more regulatory players, we will be able to further improve our mechanistic knowledge about how the AP-patterning network operates in space and time at an even finer resolution and precision.

\section{Methods}

Plasmids and lines of cells and flies. Plasmids were constructed with standard DNA-cloning methods. To express proteins in Drosophila S2 cells and HEK293T cells, the corresponding cDNA sequences were cloned into the pAc5.1/V5-His C and pcDNA3 vectors, respectively. The following plasmids were from our previous studies $^{10,13}$ : FY441 expressing HA-Bcd from the pGEM3 vector, FY442 expressing HA-Bcd from the pAc5.1/V5-His C vector and ljb3087 expressing HA-Bcd from the pcDNA3 vector. The sequence encoding FLAG-Dmpd was PCR-amplified from a $d m p d$ cDNA-containing clone (GM04147; the Drosophila Genomics Resource Center) with the following primers: $5^{\prime}$-GACTACAAGGACGACGATG ACAAGATGGTGTCCACCCGCCAAATG-3' (FLAG-Dmpd primer) and $5^{\prime}$-TGCTCTAGATTATTTACTGCGTTTGCCGGG-3'. The PCR product was digested with $\mathrm{XbaI}$ and ligated with the pGEM3 vector derived from FY441 that had been digested with $\mathrm{NcoI}$, treated with the Klenow fragment of DNA polymerase I and digested subsequently with $\mathrm{XbaI}$. The resulting intermediate plasmid, ljb4048, was then digested with HindIII and XbaI to yield a fragment (encoding FLAG-Dmpd) that was ligated with the pAC5.1/V5-His C and pcDNA3 vectors derived from HindIII and XbaI digestions of FY442 and ljb3087, respectively. The resulting plasmids ljb4049 and ljb4102 were used to express FLAG-Dmpd in Drosophila S2 and HEK293T cells, respectively. To obtain the plasmid (lib4096) expressing HA-tagged Dmpd protein (HA-Dmpd) in Drosophila S2 cells, the same cloning strategy for ljb4049 was used, except for the use of an HA-tagged Dmpd primer (5'-GCTTACCCATACGATGTTCCAGATTACGCTAT GGTGTCCACCCGCCAAATG-3') in place of the FLAG-Dmpd primer.

Drosophila S2 cells and HEK293T cells were grown in Schneider's Drosophila medium (Invitrogen) at room temperature and Dulbecco's modification of Eagle's medium (Cellgro) at $37^{\circ} \mathrm{C}$, respectively. Both media contained $10 \%(\mathrm{v} / \mathrm{v})$ fetal calf serum (Invitrogen) and $1 \times$ antibiotic-antimycotic (Invitrogen). DNA transfection into both cells was performed using the FuGENE HD transfection reagent (Roche) according to the manufacturer's instructions.

Fly lines used for obtaining embryos with different $h b$ gene copies were as follows: $D f(3 R) p 13$, red $^{1}, e^{1} / T M 3$, $S b^{1}$ (for $1 \times h b$ embryos; Bloomington Drosophila Stock 1943); $w^{1118}$ (for $2 \times h b$ embryos); KG-W25 (for $4 \times h b$ embryos; a line kindly provided by Dr James W. Posakony ${ }^{45}$ ). For the identification of $1 \times h b$ embryos, we introduced a marked balancer chromosome (TM3, hb-lacZ, which was derived from line no. 76 of the Bloomington Drosophila Stock Center) into the deficiency line to establish the $D f(3 R) p 13$, red ${ }^{1}, e^{1} / T M 3, h b-l a c Z$, $S b^{1}, \operatorname{Ser}^{1}$ flies. When these male flies were crossed to $w^{1118}$ virgin female flies, the desired $1 \times h b$ embryos were identified by a lack of lac $Z$ products. Female flies that contain different $b c d$ gene copy numbers were as follows: $S p / C y O ; b c d^{E 1} / T M 6 B$ (for $1 \times b c d$ embryos); $w^{1118}$ (for $2 \times b c d$ embryos); Sp/CyO-bcd ${ }^{+} ; \mathrm{Dr} / \mathrm{TM} 6 \mathrm{~B}$ (for $3 \times b c d$ embryos; derived from a line kindly provided by Dr Gary Struhl ${ }^{4}$ ). The fly line $d m p d^{E Y 08992}$ (Bloomington Drosophila Stock 17518) was used in P-element-mediated imprecise excision to obtain the deletion allele $d m p d^{6-3}$. The fly line $w^{1118}$; Df(2R)BSC303/Cyo (Bloomington Drosophila Stock 23686) is a deficiency line covering the entire $d m p d$-coding region.
Immunocytochemistry and reporter assay. Immunocytochemistry was per formed ${ }^{10}$ by first transfecting the HA-Dmpd-expressing plasmid into Drosophila S2 cells on a Lab-Tek II chamber slide. After fixing by $4 \%$ formaldehyde for $15 \mathrm{~min}$ at room temperature (RT), cells were permeabilized by $0.3 \%$ Triton X-100 and incubated with anti-HA antibody (1:100 dilution) at $4{ }^{\circ} \mathrm{C}$ overnight. After washing, Alexa Fluor488 goat anti-mouse IgG (1:400 dilution) was added to cells and incubated for $2 \mathrm{~h}$ at RT. TO-PRO-3 dye $(1 \mu \mathrm{M})$ was added to stain DNA for $15 \mathrm{~min}$ at RT and, after washing, images were taken under confocal microscopy.

Reporter assays were performed ${ }^{70}$ by first transfecting Drosophila S2 cells with the $h b$-CAT reporter plasmid, the HA-Bcd-expressing plasmid, the copia-lac $Z$ internal control plasmid, along with the FLAG-Dmpd-expressing plasmid (or pAc5.1/V5-His C as control). After $40 \mathrm{~h}$ post transfection, cells were harvested and lysed in $0.5 \%$ Triton X-100 by three freeze-thaw cycles. The lysates were added to a $150-\mu \mathrm{l}$ mixture containing 0.2 M Tris- $\mathrm{HCl}, \mathrm{pH} 7.5,1.6 \mathrm{mM}$ acetyl CoA (Sigma) and $0.25 \mu \mathrm{Ci}{ }^{14} \mathrm{C}$-Chloramphenicol (PerkinElmer), and incubated for $1 \mathrm{~h}$ at $37^{\circ} \mathrm{C}$. The amount of the lysate mixture used was adjusted by the transfection efficiency, which was evaluated by the $\beta$-galactosidase activity in the lysate. The products were extracted by adding $1 \mathrm{ml}$ ethyl acetate, dried by a Speed Vac Concentrator and resuspended in $20 \mu \mathrm{l}$ ethyl acetate. Ten microlitres of products were spotted in a plate for thin-layer chromatography (TLC) and allowed to migrate up the TLC plate with the chloroform: methanol (95:5) solvent. After being air-dried, the TLC plate was exposed to a storage phosphor screen (Molecular Dynamics) overnight. The screen was then scanned by the Storm 860 imaging scanner (Amersham) and intensities were quantified using the ImageQuant software.

co-IP and western blots. co-IP was performed ${ }^{10}$ in HEK293T cells that had been co-transfected with plasmids expressing HA-Bcd and FLAG-Dmpd. The transfected cells were collected and lysed in the whole-cell extract buffer $(400 \mathrm{mM}$ $\mathrm{KCl}, 10 \mathrm{mM} \mathrm{Na}_{2} \mathrm{HPO}_{4}, 1 \mathrm{mM}$ EDTA, $1 \mathrm{mM}$ dithiothreitol, $10 \%$ glycerol, $1 \mathrm{mM}$ phenylmethylsulfonyl fluoride and $0.5 \%$ NP-40 with complete protease inhibitor cocktail). Appropriate antibodies and protein G Sepharose 4 fast flow beads were added to the extracts and mixtures were incubated overnight at $4^{\circ} \mathrm{C}$. After washing with the whole-cell extract buffer, the beads were boiled in $1 \times$ SDS-PAGE loading buffer (50 mM Tris- $\mathrm{HCl}, \mathrm{pH}$ 6.8, $100 \mathrm{mM}$ dithiothreitol, 2\% SDS, 10\% glycerol and $0.1 \%$ bromophenol blue). Samples were loaded and separated using SDS-PAGE and transferred to Immun-Blot PVDF membrane (Bio-Rad). The membrane was then incubated with appropriate primary antibodies (1:1,000 dilution) followed by horseradish peroxidase-conjugated secondary antibodies (GE Healthcare, 1:2,000 dilution). Protein signals were visualized by the ECL plus western blotting detection reagents (GE Healthcare). HA-tagged and FLAG-tagged proteins were immunoprecipitated and detected by anti-HA (Covance) and anti-FLAG (Sigma) antibodies, respectively. Uncropped gel scans for western blot results are shown in Supplementary Fig. S13.

Imaging of stained embryos and data analysis. All embryos used in this study were collected at $25^{\circ} \mathrm{C}$. Procedures of FISH to detect mRNA ( $h b, l a c Z$ and eve), immunostaining to detect the Bcd protein and intron staining to detect $h b$ nascent transcripts in the fixed embryos were as follows ${ }^{10,11,44}$. Fixed embryos were hydrated stepwise to PBST buffer and post-fixed by $10 \%$ formaldehyde for $20 \mathrm{~min}$. After washing with PBST, embryos were transferred to the Hb-B buffer (50\% formamide, $5 \times$ SSC, $0.3 \%$ SDS and $0.1 \%$ Tween 20 ) stepwise and incubated with Hb-A buffer ( $50 \%$ formamide, $5 \times$ SSC, $0.3 \%$ SDS, $0.1 \%$ Tween $20,50 \mu \mathrm{g} \mathrm{ml}^{-1}$ heparin, $10 \mu \mathrm{g} \mathrm{ml}^{-1}$ yeast tRNA and $100 \mu \mathrm{g} \mathrm{ml}^{-1}$ denatured salmon sperm DNA) containing the appropriated Digoxigenin-11-UTP labelled RNA probes at $60^{\circ} \mathrm{C}$ overnight. For anti-Bcd immunostaining, the overnight incubation at $60{ }^{\circ} \mathrm{C}$ was carried out in Hb-B buffer. The embryos were transferred back stepwise to PBST buffer at $60^{\circ} \mathrm{C}$ and washed with PBST at RT for five times, followed by incubations first with $1.75 \times$ blocking buffer (Roche) for a half hour and then with the primary antibodies (1:300 dilution of anti-Digoxigenin monoclonal antibody (Roche) for FISH and intron staining or 1:100 dilution of anti-Bcd antibodies (Santa Cruz Biotechnology) for immunotaining) at $4{ }^{\circ} \mathrm{C}$ overnight. After washing with PBST buffer, the embryos were incubated with $1.75 \times$ blocking buffer for half an hour and then secondary fluorescence-conjugated secondary antibodies (1:400 dilution) for $1 \mathrm{~h}$ at RT. Individual embryos were assigned to a time class according to their mean nuclear height measured from the 'lateral' side of the midsagittal images ${ }^{44}$. Images for FISH and anti-Bcd immunotaining were captured with the Zeiss Imager $\mathrm{Z} 1$ ApoTome microscope on the midsagittal plane $\mathrm{e}^{10,11}$, and those for intron staining were captured under confocal microscopy on the surface of the nuclear monolayer with serial $z$ sections ${ }^{44}$. For FISH data analyses ${ }^{10,44}$, we first extracted raw intensities from the cytoplasmic layer using a circular window of 61 pixels to scan along the dorsal edge immediately outside of the nuclear layer (basally for $h b$; apically for eve). The scanning intervals were $2 \%$ EL for $h b$ or $1 \%$ EL for eve along the AP axis of the embryo. The background used for subtraction in an individual embryo was the average intensity at a non-expression region (between the anterior domain and posterior stripe for $h b$; the anterior $10 \%$ EL domain for eve). To extract raw Bcd intensities from immunostained embryos, a circular window was used to scan the dorsal nuclear layer of the midsagittal images. To generate an intensity profile as a function of absolute distance from the anterior $(x)$ or relative AP position $(x / L)$, the scanning intervals were set as either $\sim 12 \mu \mathrm{m}$ or $2 \%$ EL. To calculate $\rho$ from intron-staining data, all nuclei in an individual embryo were 
grouped into bins of the size of $2 \% \mathrm{EL}$ along the AP axis. The average intron dot number per nucleus within each bin was calculated and defined as $\rho$. The measurement of EL required stitching two sets of images that were captured separately for the anterior and posterior parts of an embryo. The anterior $\sim 60 \%$ EL domain for each embryo (where the intron-staining data were used in $\rho$ calculations) was captured by a single set of images. To calculate the length constant $(\lambda)$ of the Bcd gradient profile from an individual embryo, the equation of $B=A \times \mathrm{e}^{-x / \lambda}+C$ was used to fit the experimentally extracted data ${ }^{10}$. To avoid data from the non-exponential anterior region and the error-prone posterior region, fitting was performed within the $x / L$ range of $0.2-0.9$. Data fitting and other statistical analyses including Student's $t$-tests (two-tailed) were performed using the Matlab software (MathWorks).

\section{References}

1. Driever, W. \& Nusslein-Volhard, C. A gradient of bicoid protein in Drosophila embryos. Cell 54, 83-93 (1988).

2. Ephrussi, A. \& St Johnston, D. Seeing is believing: the bicoid morphogen gradient matures. Cell 116, 143-152 (2004).

3. Driever, W. \& Nusslein-Volhard, C. The bicoid protein is a positive regulator of hunchback transcription in the early Drosophila embryo. Nature 337, 138-143 (1989).

4. Struhl, G., Struhl, K. \& Macdonald, P. M. The gradient morphogen bicoid is a concentration-dependent transcriptional activator. Cell 57, 1259-1273 (1989).

5. Bender, M., Horikami, S., Cribbs, D. \& Kaufman, T. C. Identification and expression of the gap segmentation gene hunchback in Drosophila melanogaster. Dev. Genet. 9, 715-732 (1988).

6. Tautz, D. et al. Finger protein of novel structure encoded by hunchback, a 2nd member of the gap class of Drosophila segmentation genes. Nature 327, 383-389 (1987)

7. Driever, W. \& Nusslein-Volhard, C. The bicoid protein determines position in the Drosophila embryo in a concentration-dependent manner. Cell 54, 95-104 (1988).

8. Berleth, T. et al. The role of localization of bicoid RNA in organizing the anterior pattern of the Drosophila embryo. EMBO. J. 7, 1749-1756 (1988).

9. Liu, J., He, F. \& Ma, J. Morphogen gradient formation and action: insights from studying Bicoid protein degradation. Fly (Austin) 5, 242-246 (2011).

10. Liu, J. \& Ma, J. Fates-shifted is an F-box protein that targets Bicoid for degradation and regulates developmental fate determination in Drosophila embryos. Nat. Cell. Biol. 13, 22-29 (2011).

11. He, F. et al. Probing intrinsic properties of a robust morphogen gradient in Drosophila. Dev. Cell 15, 558-567 (2008).

12. Schaeffer, V., Janody, F., Loss, C., Desplan, C. \& Wimmer, E. A. Bicoid functions without its TATA-binding protein-associated factor interaction domains. Proc. Natl Acad. Sci. USA 96, 4461-4466 (1999).

13. Zhao, C. et al. The activity of the Drosophila morphogenetic protein Bicoid is inhibited by a domain located outside its homeodomain. Development 129, 1669-1680 (2002)

14. Liu, J. \& Ma, J. Drosophila Bicoid is a substrate of sumoylation and its activator function is subject to inhibition by this post-translational modification. FEBS Lett. 586, 1719-1723 (2012).

15. Driever, W., Ma, J., Nusslein-Volhard, C. \& Ptashne, M. Rescue of bicoid mutant Drosophila embryos by bicoid fusion proteins containing heterologous activating sequences. Nature 342, 149-154 (1989).

16. Simpson-Brose, M., Treisman, J. \& Desplan, C. Synergy between the hunchback and bicoid morphogens is required for anterior patterning in Drosophila. Cell 78, 855-865 (1994).

17. Arnosti, D. N., Barolo, S., Levine, M. \& Small, S. The eve stripe 2 enhancer employs multiple modes of transcriptional synergy. Development 122, 205-214 (1996)

18. Ho, M. S., Tsai, P. I. \& Chien, C. T. F-box proteins: the key to protein degradation. J. Biomed. Sci. 13, 181-191 (2006).

19. Cardozo, T. \& Pagano, M. The SCF ubiquitin ligase: insights into a molecular machine. Nat. Rev. Mol. Cell Biol. 5, 739-751 (2004).

20. Skaar, J. R., Pagan, J. K. \& Pagano, M. SnapShot: F box proteins I. Cell 137, 1160-1160 e1161 (2009)

21. Skaar, J. R., D’Angiolella, V., Pagan, J. K. \& Pagano, M. SnapShot: F box proteins II. Cell 137, 1358, e1351 (2009).

22. Deshaies, R. J. SCF and Cullin/Ring H2-based ubiquitin ligases. Annu. Rev. Cell Dev. Biol. 15, 435-467 (1999).

23. Freed, E. et al. Components of an SCF ubiquitin ligase localize to the centrosome and regulate the centrosome duplication cycle. Genes Dev. 13, 2242-2257 (1999)

24. Seol, J. H. et al. Cdc53/cullin and the essential Hrt1 RING-H2 subunit of SCF define a ubiquitin ligase module that activates the E2 enzyme Cdc34. Genes Dev. 13, 1614-1626 (1999).

25. Muratani, M., Kung, C., Shokat, K. M. \& Tansey, W. P. The F box protein Dsg1/Mdm30 is a transcriptional coactivator that stimulates Gal4 turnover and cotranscriptional mRNA processing. Cell 120, 887-899 (2005).
26. Conaway, R. C., Brower, C. S. \& Conaway, J. W. Emerging roles of ubiquitin in transcription regulation. Science 296, 1254-1258 (2002).

27. Lipford, J. R. \& Deshaies, R. J. Diverse roles for ubiquitin-dependent proteolysis in transcriptional activation. Nat. Cell Biol. 5, 845-850 (2003).

28. von der Lehr, N. et al. The F-box protein Skp2 participates in c-Myc proteosomal degradation and acts as a cofactor for c-Myc-regulated transcription. Mol. Cell 11, 1189-1200 (2003).

29. Kim, S. Y., Herbst, A., Tworkowski, K. A., Salghetti, S. E. \& Tansey, W. P. Skp2 regulates Myc protein stability and activity. Mol. Cell 11, 1177-1188 (2003).

30. Kimbrel, E. A. \& Kung, A. L. The F-box protein beta-TrCp1/Fbwla interacts with p300 to enhance beta-catenin transcriptional activity. J. Biol. Chem. 284 13033-13044 (2009).

31. Hsu, T., McRackan, D., Vincent, T. S. \& Gert de Couet, H. Drosophila Pin1 prolyl isomerase Dodo is a MAP kinase signal responder during oogenesis. Nat. Cell. Biol. 3, 538-543 (2001).

32. Ma, X., Yuan, D., Diepold, K., Scarborough, T. \& Ma, J. The Drosophila morphogenetic protein Bicoid binds DNA cooperatively. Development 122, 1195-1206 (1996).

33. Yuan, D., Ma, X. \& Ma, J. Recognition of multiple patterns of DNA sites by Drosophila homeodomain protein Bicoid. J. Biochem. 125, 809-817 (1999).

34. Sadowski, I., Ma, J., Triezenberg, S. \& Ptashne, M. GAL4-VP16 is an unusually potent transcriptional activator. Nature 335, 563-564 (1988).

35. Crauk, O. \& Dostatni, N. Bicoid determines sharp and precise target gene expression in the Drosophila embryo. Curr. Biol. 15, 1888-1898 (2005).

36. Perry, M. W., Bothma, J. P., Luu, R. D. \& Levine, M. Precision of hunchback expression in the Drosophila embryo. Curr. Biol. 22, 2247-2252 (2012).

37. Bergmann, S. et al. Pre-steady-state decoding of the Bicoid morphogen gradient. PLoS Biol. 5, e46 (2007).

38. Houchmandzadeh, B., Wieschaus, E. \& Leibler, S. Establishment of developmental precision and proportions in the early Drosophila embryo. Nature 415, 798-802 (2002).

39. Jaeger, J. et al. Dynamic control of positional information in the early Drosophila embryo. Nature 430, 368-371 (2004).

40. Gregor, T., Tank, D. W., Wieschaus, E. F. \& Bialek, W. Probing the limits to positional information. Cell 130, 153-164 (2007).

41. Jaeger, J. The gap gene network. Cell. Mol. Life Sci. 68, 243-274 (2011).

42. Chen, H., Xu, Z., Mei, C., Yu, D. \& Small, S. A system of repressor gradients spatially organizes the boundaries of bicoid-dependent target genes. Cell 149, 618-629 (2012).

43. He, F., Ren, J., Wang, W. \& Ma, J. Evaluating the Drosophila Bicoid morphogen gradient system through dissecting the noise in transcriptional bursts. Bioinformatics 28, 970-975 (2012).

44. Liu, J. \& Ma, J. Uncovering a dynamic feature of the transcriptional regulatory network for anterior-posterior patterning in the Drosophila embryo. PLoS One 8, e62641 (2013).

45. Margolis, J. S. et al. Posterior stripe expression of hunchback is driven from two promoters by a common enhancer element. Development 121, 3067-3077 (1995).

46. Struhl, G., Johnston, P. \& Lawrence, P. A. Control of Drosophila body pattern by the hunchback morphogen gradient. Cell 69, 237-249 (1992).

47. Payankaulam, S. \& Arnosti, D. N. Gene regulation: boundaries within limits. Curr. Biol. 18, R653-R655 (2008).

48. Yu, D. \& Small, S. Precise registration of gene expression boundaries by a repressive morphogen in Drosophila. Curr. Biol. 18, 868-876 (2008).

49. Bothma, J. P., Magliocco, J. \& Levine, M. The snail repressor inhibits release, not elongation, of paused Pol II in the Drosophila embryo. Curr. Biol. 21, 1571-1577 (2011).

50. Ma, J. Crossing the line between activation and repression. Trends Genet. 21, 54-59 (2005).

51. Geng, F., Wenzel, S. \& Tansey, W. P. Ubiquitin and proteasomes in transcription. Annu. Rev. Biochem. 81, 177-201 (2012).

52. Kodadek, T., Sikder, D. \& Nalley, K. Keeping transcriptional activators under control. Cell 127, 261-264 (2006).

53. Muratani, M. \& Tansey, W. P. How the ubiquitin-proteasome system controls transcription. Nat. Rev. Mol. Cell Biol. 4, 192-201 (2003).

54. Wartlick, O., Kicheva, A. \& Gonzalez-Gaitan, M. Morphogen gradient formation. Cold Spring Harb. Perspect. Biol. 1, a001255 (2009).

55. Dui, W., Lu, W., Ma, J. \& Jiao, R. A systematic phenotypic screen of F-box genes through a tissue-specific RNAi-based approach in Drosophila. J. Genet. Genomics 39, 397-413 (2012).

56. Deng, J., Wang, W., Lu, L. J. \& Ma, J. A two-dimensional simulation model of the bicoid gradient in Drosophila. PLoS One 5, e10275 (2010).

57. Cheung, D., Miles, C., Kreitman, M. \& Ma, J. Scaling of the Bicoid morphogen gradient by a volume-dependent production rate. Development 138, 2741-2749 (2011). 
58. Cheung, D., Miles, C., Kreitman, M. \& Ma, J. Adaptation of the length scale and amplitude of the Bicoid gradient profile to achieve robust patterning in abnormally large Drosophila melanogaster embryos. Development doi:10.1242/dev.098640 (2013).

59. Porcher, A. et al. The time to measure positional information: maternal hunchback is required for the synchrony of the Bicoid transcriptional response at the onset of zygotic transcription. Development 137, 2795-2804 (2010).

60. Foe, V. E. \& Alberts, B. M. Studies of nuclear and cytoplasmic behaviour during the five mitotic cycles that precede gastrulation in Drosophila embryogenesis. J. Cell Sci. 61, 31-70 (1983).

61. He, F. \& Ma, J. A spatial point pattern analysis in Drosophila blastoderm embryos evaluating the potential inheritance of transcriptional states. PLoS One 8, e60876 (2013).

62. Shermoen, A. W. \& O'Farrell, P. H. Progression of the cell cycle through mitosis leads to abortion of nascent transcripts. Cell 67, 303-310 (1991).

63. Lagha, M. et al. Paused Pol II coordinates tissue morphogenesis in the Drosophila embryo. Cell 153, 976-987 (2013).

64. Lucas, T. et al. Live imaging of Bicoid-dependent transcription in Drosophila embryos. Curr. Biol. 23, 2135-2139 (2013).

65. Garcia, H. G., Tikhonov, M., Lin, A. \& Gregor, T. Quantitative imaging of transcription in living Drosophila embryos links polymerase activity to patterning. Curr. Biol. 23, 2140-2145 (2013).

66. Bertrand, E. et al. Localization of ASH1 mRNA particles in living yeast. Mol. Cell 2, 437-445 (1998).

67. Forrest, K. M. \& Gavis, E. R. Live imaging of endogenous RNA reveals a diffusion and entrapment mechanism for nanos mRNA localization in Drosophila. Curr. Biol. 13, 1159-1168 (2003).

68. Little, S. C., Tikhonov, M. \& Gregor, T. Precise developmental gene expression arises from globally stochastic transcriptional activity. Cell 154, 789-800 (2013).
69. He, F., Ren, J., Wang, W. \& Ma, J. A multiscale investigation of bicoiddependent transcriptional events in Drosophila embryos. PLoS One 6, e19122 (2011).

70. Zhao, C., Dave, V., Yang, F., Scarborough, T. \& Ma, J. Target selectivity of Bicoid is dependent on non-consensus site recognition and protein-protein interaction. Mol. Cell Biol. 20, 8112-8123 (2000).

\section{Acknowledgements}

We thank members of our groups at CCHMC for discussion and assistance. We also thank the Bloomington Stock Center, Dr. James W. Posakony and Dr Gary Struhl for fly lines. This work was supported in part by grants from NIH (R01GM101373) and NSF (IOS-0843424) to J.M.

\section{Author contributions}

J.L. and J.M. conceived and designed the study; J.L. performed all experiments and analyses; J.L. and J.M. analysed data; J.L. generated all figures; J.L. and J.M. wrote and approved the paper.

\section{Additional information}

Supplementary Information accompanies this paper at http://www.nature.com/ naturecommunications

Competing financial interests: The authors declare no competing financial interests.

Reprints and permission information is available online at http://npg.nature.com/ reprintsandpermissions/

How to cite this article: Liu, J. \& Ma, J. Dampened regulates the activating potency of Bicoid and the embryonic patterning outcome in Drosophila. Nat. Commun. 4:2968 doi: $10.1038 /$ ncomms3968 (2013) 